website extra

Additional information

appears on the

Evidence-Based

Mental Health

website

www.ebmentalhealth. com

Source of funding: no external funding.

For correspondence: $\mathrm{Dr}$ D S Knopman,

Department of

Clinic, 200 First St SW

Rochester, MN, 55905 ,

USA.

knopman@

maroon.tc.umn.edu.

Diagnosis

\title{
Review: most laboratory tests do not add to the diagnostic accuracy of clinical criteria for dementia
}

Knopman DS, DeKosky ST, Cummings JL, et al. Practice parameter: diagnosis of dementia (an evidence-based review). Report of the Quality Standards Subcommittee of the American Academy of Neurology. Neurology 2001 May 8;56:1143-53.

QUESTIONS: Are current diagnostic criteria accurate for diagnosing prevalent dementias in the elderly? Do laboratory tests improve the accuracy of the clinical diagnosis of dementia?

Neurology, Mayo

\section{Data sources}

Studies were identified by searching electronic databases and reference lists and contacting experts.

\section{Study selection}

English language studies on the diagnosis of dementia in humans were selected. Studies on Alzheimer's disease (AD) were selected if they included $>25$ patients.

\section{Data extraction}

The quality of studies was rated. Data were extracted on diagnostic precision.

\section{Main results}

Clinical criteria: the table shows the results. Laboratory tests: a study showed that a minimum width of the medial temporal lobe below the $5^{\text {th }}$ percentile was $95 \%$ sensitive but $40 \%$ specific for detecting $\mathrm{AD}$. In 5 studies of medial temporal lobe atrophy, sensitivity ranged from $77-92 \%$ and

Diagnostic accuracy of criteria for assessing dementia*

\begin{tabular}{|c|c|c|c|c|}
\hline Outcomes & Criteria & $\begin{array}{l}\text { Number } \\
\text { of studies }\end{array}$ & $\begin{array}{l}\text { Mean } \\
\text { sensitivity } \\
\text { (range) }\end{array}$ & $\begin{array}{l}\text { Mean } \\
\text { specificity } \\
\text { (range) }\end{array}$ \\
\hline DAT & $D S M-I I I-R$ & 3 & $81 \%(49-100)$ & $70 \%(47-100)$ \\
\hline Probable AD & NINCDS-ADRDA & 10 & & \\
\hline Possible AD & & 4 & $93 \%(85-96)$ & $48 \%(32-61)$ \\
\hline \multirow[t]{2}{*}{ Vascular dementia } & NINDS-AIREN & 1 & $43 \%$ & $95 \%$ \\
\hline & $\begin{array}{l}\text { HIS/DSM-III } \\
\text { R/NINDS-AIREN/California }\end{array}$ & 4 & $50 \%(20-89)$ & $87 \%(64-98)$ \\
\hline $\begin{array}{l}\text { Dementia with Lewy } \\
\text { bodies (DLB) }\end{array}$ & $\begin{array}{l}\text { Consortium for DLB } \\
\text { diagnostic criteria }\end{array}$ & 1 & $\begin{array}{l}22 \% \\
58 \%(34-75)\end{array}$ & $\begin{array}{l}100 \% \\
87 \%(71-94)\end{array}$ \\
\hline
\end{tabular}

*DAT=Dementia of the Alzheimer type; AD=Alzheimer's disease; HIS=Hachinski Ischemic Score. specificity from $49-95 \%$ for detecting AD. The sensitivity for single-photon emission computed tomography (SPECT) was not higher than that for clinical criteria. For differentiating $\mathrm{AD}$ from non- $\mathrm{AD}$ dementia, 2 studies showed sensitivities of $86 \%$ and $95 \%$ and specificities of $42 \%$ and $73 \%$. Positron emission tomography (PET) had a sensitivity of $93 \%$ and specificity of $63 \%$ for detecting $\mathrm{AD}$ in 1 study. PET had better diagnostic accuracy than SPECT for detecting AD (87.2\% v 62.9\% for Mini-Mental State Examination [MMSE] score $>20$ and 100\% $v$ 81.2\% for MMSE score <20). Genetic biomarkers: 1 study showed that the presence of apoliprotein E (APOE) $\varepsilon 4$ increased the positive predictive value of the $\mathrm{AD}$ diagnosis from $90 \%$ to $94 \%$. In patients with non- $\mathrm{AD}$, the absence of APOE $\varepsilon 4$ increased the negative predictive value from $64 \%$ to $72 \%$. Cerebrospinal fluid (CSF) markers: studies showed that the CSF markers $\left(\beta\right.$ amyloid $_{\mathrm{I}-42}$, tau, and $\mathrm{AD} 7 \mathrm{C}$ protein) have moderate to high sensitivity and specificity for detecting $\mathrm{AD}$, but it is unclear whether their combined use is better than a competent clinical diagnosis. 2 studies showed that an immunoassay for the detection of the 14-3-3 protein in CSF had a sensitivity of $99 \%$ and $94 \%$ and a specificity of $96 \%$ and $93 \%$ for detecting Creutzfeldt-Jakob disease.

\section{Conclusions}

Clinical criteria for probable Alzheimer's disease, dementia of the Alzheimer type, and Creutzfeldt-Jakob disease (CJD) have diagnostic accuracy sufficient for routine use. The cerebrospinal fluid 14-3-3 protein assay is useful for confirming the diagnosis of CJD. No other laboratory test improves diagnostic accuracy.

\section{COMMENTARY}

Dementia, a common mental health syndrome, affects approximately $5-10 \%$ of people $\geqslant 65$ years of age. This diagnosis has important implications for patients, their families, and society in general. Hence, clinicians who care for older adults should be fully aware of currently available diagnostic criteria, their reliability and validity, and which tests may improve diagnostic accuracy.

The American Academy of Neurology has put together a distinguished group of professionals to systematically review recent advances in the diagnosis of dementia. The Committee concluded that the DSM-III-R, DSM-IV and NINCDS-ADRDA criteria for dementia have good reliability. The authors did not describe any studies testing the reliability of the $I C D-10$ definition of dementia. This omission is unfortunate because the $I C D-10$ is used internationally.

The Committee further concluded that the NINCDS-ADRDA or DSM-III-R criteria for AD should be used routinely, even though a high sensitivity for the diagnosis is often achieved at the expense of low specificity. NINCDS-AIREN and DSM-III-R criteria for vascular dementia lack sensitivity and, in clinical practice, the Hachinski Ischaemic Score may be more useful. Currently available criteria for dementia with Lewy bodies (DLB) fail to reliably discriminate DLB from AD. The Lund-Manchester criteria for frontotemporal dementia seem to have good sensitivity and specificity, but information is sparse.

Structural or functional neuroimaging fails to significantly improve the accuracy of the clinical diagnosis, although structural MRI or CT is recommended as part of the initial evaluation of patients with dementia. Genetic testing and CSF markers are not appropriate for routine use (except for the 14-3-3 protein for CJD). The Committee recommended that all patients who are assessed for dementia should be screened for the presence of depression, vitamin B12 deficiency, and hypothyroidism. This advice may be controversial because the prevalence of B12 deficiency and hypothyroidism in dementia services is relatively low and unlikely to be the cause of dementia-screening for folate deficiency may be more clinically relevant.

This review provides a wealth of information on issues related to the diagnosis of dementia that will be useful to clinicians and researchers. However, it is disappointing to note that we have not gained much ground since the practice parameter was first published in 1994.

Osvaldo P Almeida University of Western Australia; Perth, Western Australia, Australia 